\title{
Survival With Emergency Tourniquet Use to Stop Bleeding in Major Limb Trauma
}

\author{
COL John F. Kragh, Jr., MC, USA, * Thomas J. Walters, PhD, * David G. Baer, PhD, * \\ LTC Charles J. Fox, MC, USA, † Charles E. Wade, PhD, * Jose Salinas, PhD,* \\ and COL John B. Holcomb, MC, USA*
}

\begin{abstract}
Objective: The purpose of this study was to determine if emergency tourniquet use saved lives.
\end{abstract}

Summary Background Data: Tourniquets have been proposed as lifesaving devices in the current war and are now issued to all soldiers. Few studies, however, describe their actual use in combat casualties.

Methods: A prospective survey of injured who required tourniquets was performed over 7 months in 2006 (NCT00517166 at ClinicalTrials.gov). Follow-up averaged 28 days. The study was at a combat support hospital in Baghdad. Among 2838 injured and admitted civilian and military casualties with major limb trauma, $232(8 \%)$ had 428 tourniquets applied on 309 injured limbs. We looked at emergency tourniquet use, and casualties were evaluated for shock (weak or absent radial pulse) and prehospital versus emergency department (ED) tourniquet use. We also looked at those casualties indicated for tourniquets but had none used. We assessed survival rates and limb outcome.

Results: There were 31 deaths (13\%). Tourniquet use when shock was absent was strongly associated with survival $(90 \%$ vs. $10 \% ; P<0.001)$. Prehospital tourniquets were applied in 194 patients of which 22 died (11\% mortality), whereas 38 patients had ED application of which 9 died $(24 \%$ mortality; $P=$ 0.05 ). The 5 casualties indicated for tourniquets but had none used had a survival rate of $0 \%$ versus $87 \%$ for those casualties with tourniquets used $(P<0.001)$. Four patients $(1.7 \%)$ sustained transient nerve palsy at the level of the tourniquet. No amputations resulted solely from tourniquet use.

Conclusions: Tourniquet use when shock was absent was strongly associated with saved lives, and prehospital use was also strongly associated with lifesaving. No limbs were lost due to tourniquet use. Education and fielding of prehospital tourniquets in the military environment should continue.

(Ann Surg 2009;249: 1-7)

From the *US Army Institute of Surgical Research, Fort Sam Houston, TX; and $\dagger$ Walter Reed Army Medical Centre, Georgia Avenue North West, Washington DC.

Study performed at 10th Combat Support Hospital, US Army Task Force North, APO AE 09348 (Ibn Sina Hospital, International Zone, Baghdad, Iraq).

COL Kragh conceived and designed the work, collected the data, analyzed data, and produced the article. Drs Walters and Baer participated in conception, design, and writing. MAJ Fox, Dr Wade, and COL Holcomb participated in data collection, analysis, and writing. Dr Salinas participated in design, analysis, and writing. Drs Wade, Baer, and COL Kragh participated in the regulatory oversight.

The funding of this work was only for the general salary of the investigators in the course of their federal employment. There was no sponsor and the authors declare no conflicts of interest.

No reprints are available from the authors. There was no grant; the work was supported by US Army internal funds. We consult at no cost with tourniquet companies that engage us on design improvements. We have cooperative research and development agreements and material transfer agreements with such companies that protect intellectual property rights and the like.

The opinions or assertions contained herein are the private views of the authors and are not to be construed as official or reflecting the views of the Department of Defense or United States Government. The authors are employees of the US government. This work was prepared as part of their official duties and, as such, there is no copyright to be transferred.

Copyright (C) 2009 by Lippincott Williams \& Wilkins

ISSN: 0003-4932/09/24901-0001

DOI: $10.1097 /$ SLA.0b013e31818842ba
$\mathrm{H}$ emorrhage from injured extremities continues to be one of the leading sources of preventable death on the battlefield. ${ }^{1-4}$ Data from recent conflicts involving US military personnel confirmed the continued importance of improving prehospital hemorrhage control. $^{3,5-7}$ In response, the US Army implemented a design, testing, training, and fielding program for battlefield tourniquets, ${ }^{8-11}$ resulting in policy that all military personnel in theater carry tourniquets. As a result of this effort, tourniquets are now common on the battlefields of Iraq and Afghanistan, both in the hands of medical and nonmedical personnel.

With the Tactical Combat Casualty Care initiative, the US military is not alone in establishing procedures and equipment for use of tourniquets in the prehospital environment by both medical and nonmedical personnel. ${ }^{12,13}$ However, this renewed emphasis on tourniquets for prehospital hemorrhage control of extremity injuries is not agreed upon by all authors ${ }^{14-16}$ with some authors discouraging prehospital use of tourniquets altogether. ${ }^{17-20}$ Dorlac et $\mathrm{al}^{21}$ showed that tourniquet use is indicated in civilian trauma, albeit in a very small percentage of patients. However, the lifesaving capability of tourniquets has been unproven. Most of the controversy regarding the capacity of tourniquets to save lives versus tissue damage has been based more on speculation rather than actual data, as research in the human use of emergency tourniquets is limited. Clearly, the discussion would be better informed with actual data regarding these critical concerns. In 2003, we initiated data collection regarding emergency tourniquet use, and this study is a continuation and amplification of that effort. ${ }^{22}$

We performed a prospective observational study at the US combat support hospital in Baghdad, Iraq of patients who had tourniquets applied to determine if emergency tourniquet use saved lives.

\section{METHODS}

\section{Study Design}

The protocol was approved by the institutional review board, and the study was registered(NCT00517166 at ClinicalTrials. gov). The study period was from March 19 to October 4, 2006, the first author's arrival and departure dates at the study site, Ibn Sina Hospital, Baghdad, Iraq. This was a prospective observational survey with cohort and subgroup analyses. All patients at the combat support hospital who had a tourniquet of any type used in their emergent health care [prehospital, emergency department (ED), or intensive care unit (ICU)] were included in the study. Detainees and prisoners of war are restricted from research by military policies and were excluded. No experimental interventions were made, and the procedures were conducted in accord with the ethical standards of the Helsinki Declaration of 1975. The informed consent waiver was approved.

\section{Data Collection}

Data collected included patient age in years, gender, application time (time between injury and use) in minutes, setting of tourniquet application (prehospital or ED), mechanism of injury, injury type (such 
as blunt or penetrating trauma), treatment (including operative procedures, number of transfused units (all blood products were summed), injury severity scores (ISS), abbreviated injury score (AIS), base deficit, systolic blood pressure, international normalized ratio (INR), initial heart rate, injury description (eg, traumatic amputation, open fracture, artery lesion, etc.), outcome (limb salvage, death), complications (necrotic muscle, compartment syndrome, nerve palsy), and duration of follow-up. We had access to electronic records of US military casualties in Iraq, Germany, and the United States.

\section{Definitions}

A tourniquet was defined as any limb constrictive device, whether improvised or commercially manufactured, used in an attempt to stop extremity bleeding.

We evaluated tourniquet use in 2 ways: we categorized patients by where their tourniquets were placed geographicallyprehospital or in the ED and when they were placed physiologically in relation to shock. ED tourniquet patients were those patients who had a tourniquet first placed on a limb in the ED, and other patients were prehospital. We also included 1 intensive care unit patient in the ED group.

Shock was defined by medics or hospital providers as a weak or absent radial pulse in an uninjured limb without a tourniquet. Patients with tourniquets first placed after the onset of shock were analyzed as shock present, and all other patients were absent shock before application of first tourniquet. This validated approach is consistent with the clinical definitions used by the Tactical Combat Casualty Care course, taught to all military medics, and correlated with systolic blood pressures by McManus et al. ${ }^{23,24}$

\section{Statistical Analysis}

Descriptive statistics were used for tourniquet applications and resulting outcomes. For comparisons between subgroups with categorical data, significance was determined using $\chi^{2}$ or, when counts were less than 5, Fisher exact test. Subgroup analyses for continuous data were done with Student $t$ test. All tests were 2-tailed. A $P \leq 0.05$ was the critical value for determining significance for all tests except mortality for which we used a value of

TABLE 1. Mechanisms of Injury Indicating Tourniquet Use for 309 Limbs in 232 Patients

\begin{tabular}{lc}
\hline Mechanisms of Injury & Patients N* \\
\hline Explosions & 232 \\
Gunshot & 73 \\
Burn & 17 \\
Motor vehicle crash & 13 \\
Knife & 1 \\
\hline
\end{tabular}

*Patients had 1 to 4 mechanisms of injury.
$P<0.1$. In this use, standard practice uses a significance threshold of $P=0.1$ for testing of mortality to increase the likelihood of detecting a survival benefit when one was present. Survival rates over time were compared with Kaplan-Meier survivorship analysis using the log rank (Mantel-Cox) test. A series of contingency tables were used to test the association of survival to shock (present v. absent at the time of application) and prehospital versus ED tourniquet use.

\section{RESULTS}

\section{Patient Demographics}

The study group was 232 patients ( $8.2 \%$ of 2838 trauma admissions) that had 428 tourniquets applied on 309 limbs. There were 220 males and 12 females in a multinational patient population. The average patient age was 29 years (median, 28; range, 4-70). There were 9 children $(<18$-year old) and 1 elderly person $(>65$-year old). Follow-up averaged 18 days (range, $0.5-152$ days; median, 7 days). The majority of injuries (210/336 or 63\%) were due to explosions (Table 1$)$. The mean ISS was 14 (median, 10). Physiologic data are shown in Table 2 and Figure 1, and injuries are shown in Table 3. Prehospital tourniquets, when known, were applied 0 to 75 minutes after injury with an average and median of 10 minutes.

The 428 tourniquets were used on 308 limbs sometimes singly, but if bleeding continued a second or more were added side by side. Some broke, leaked, malfunctioned, or were misused, a few were replaced after removal for temporary success with stopping bleeding, and some prehospital devices were exchanged for wider, safer hospital devices. ${ }^{1}$

Patients were injured in complex events that could involve multiple mechanisms often in sequence resulting in complicated wounds. For example, some patients rode in vehicles that had roadside explosions causing blast overpressure, penetrating fragments, vehicle rollover with blunt trauma or crush injury, and then fire with burns. Superficial burns were sometimes associated with unburned deep tissues that bled, eg, a burned patient then got shot and bled. ${ }^{1}$

\section{Survival Rate Was Higher in Patients With Tourniquets Used Versus Tourniquets Not Used}

A matched subgroup of 13 patients from the 232 in the study group with tourniquets were compared with the patients who presented to the hospital without tourniquets in place but would have potentially benefited from tourniquet use (Fig. 2A, Table 4). We identified only 5 patients (3 American, 2 Iraqi) during the study period with isolated limb injuries that were compressible and thus amenable to tourniquet use.

These 5 patients met the indications for tourniquet use in the field, but the patient was extricated or transported before tourniquet use was implemented. The 5 were alive in the field, and were

TABLE 2. Pathophysiologic Data Summary

\begin{tabular}{lccccc}
\hline & N (232) & Average \pm SD & Median & Minimum & Maximum \\
\hline Injury severity scores & 210 & $14 \pm 10.5$ & 10 & 1 & 75 \\
Initial base deficit & 197 & $-7 \pm 7.1$ & -4 & -27 & 2 \\
Initial heart rate & 213 & $107 \pm 35.5$ & 110 & 0 & 208 \\
Initial systolic blood pressure (mm Hg) & 189 & $112 \pm 37.1$ & 120 & 0 & 200 \\
INR & 199 & $1.8 \pm 5.41$ & 1.2 & 0.6 & 74 \\
Transfusion units & 217 & $20 \pm 31.7$ & 8 & 0 & 194
\end{tabular}

The transfusion units are the sum of all blood products of all types.

SD indicates standard deviation; mm Hg, millimeters of Mercury; N, number of patients with data. 
A

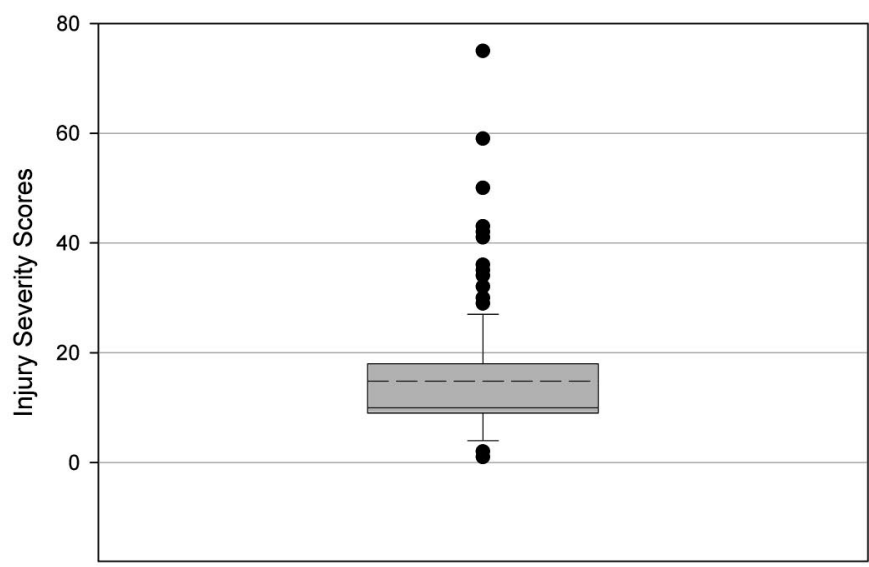

C

Initial Systolic Blood Pressure

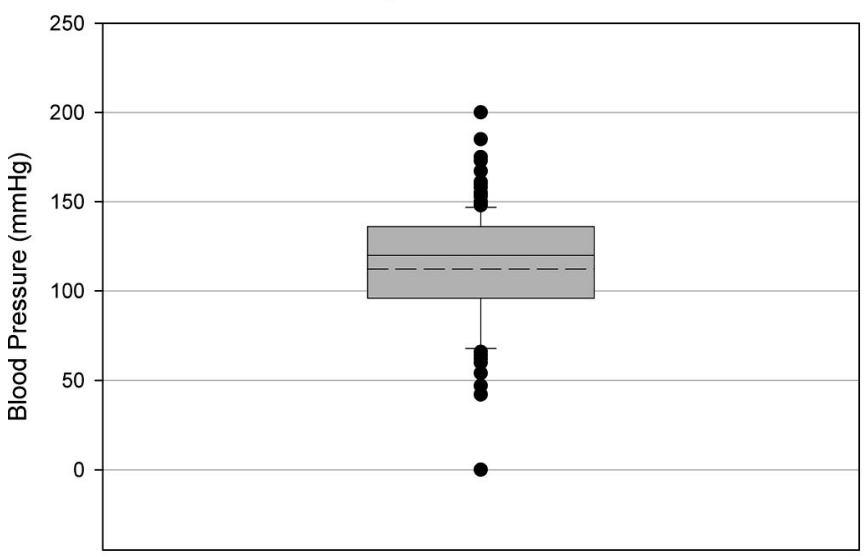

E

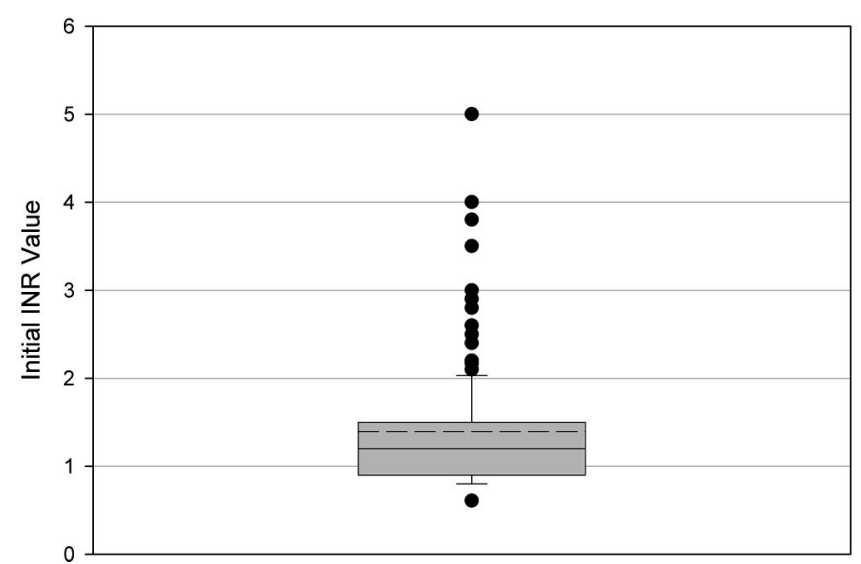

B

Initial Heart Rate

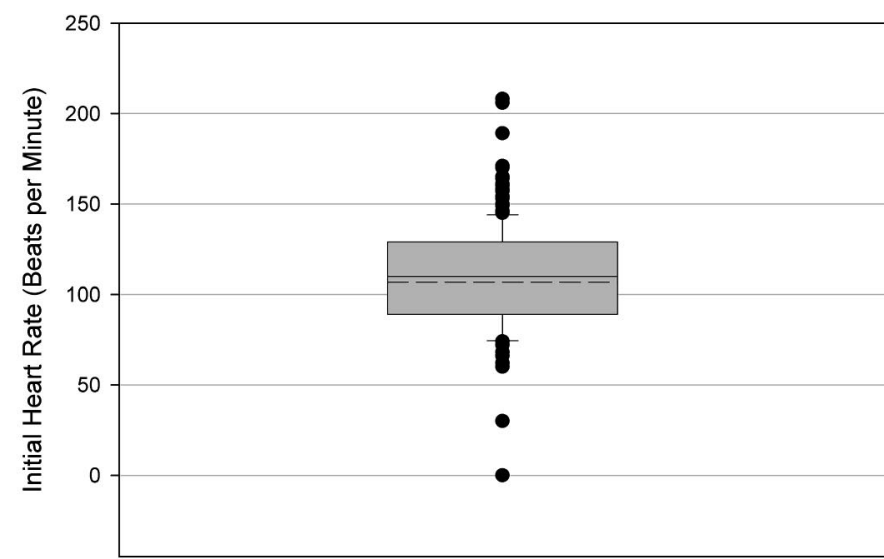

D

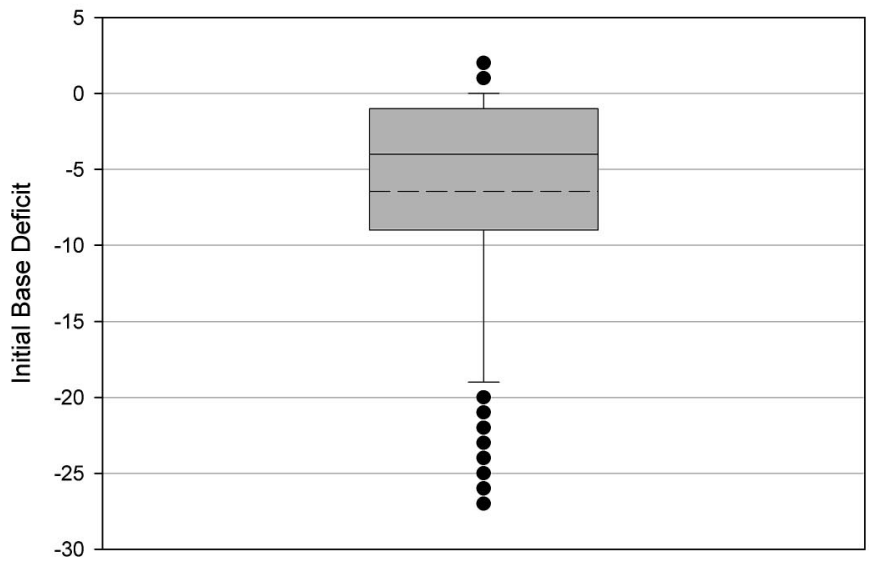

$\mathbf{F}$

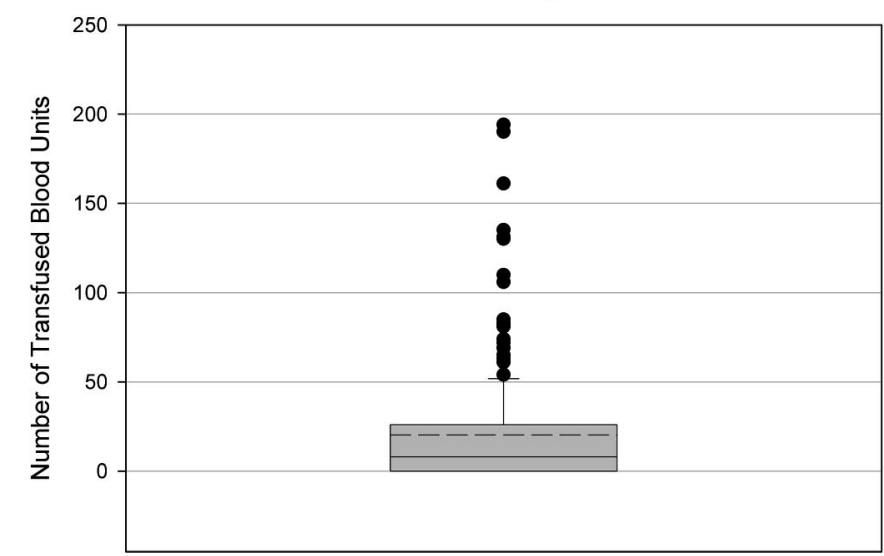

FIGURE 1. A-F, Physiologic data. Box plots of physiologic data with outliers as black dots, $25 \%$ and $75 \%$ limits as box borders, $95 \%$ confidence limits as whiskers, median as solid line, and mean as dashed line. A, Injury severity scores. B, Initial heart rate on presentation. Ten patients had initial rates of 0 , and 135 patients had tachycardia. C, Initial systolic blood pressure. D, Initial base deficit on presentation. Two patients were -27 , and 75 patients were -6 or less; a -6 value indicated substantial physiologic abnormality. E, Initial international normalized ratio (INR) on presentation. The 74 and 20.5 values were deleted from the graphic to better show the spread of the bulk of the data. For initial INR values, 1 patient was 74 and 1 patient was 20.5, and 51 patients were 1.5 or higher; a 1.5 value indicated coagulopathy. F, Number of transfused blood units. The transfusion units are the sum of all blood products of all types. A value of 10 units constituted a massive transfusion; 104 patients had massive transfusions. 
TABLE 3. Number of Injuries by Type in Cases With Tourniquet Use for 309 Limbs in 232 Patients

\begin{tabular}{lc}
\hline Types of Injuries & Number of Limbs With the Indication* \\
\hline Traumatic amputations & 97 \\
Vascular injuries & 85 \\
Open fractures & 81 \\
Soft tissue injuries & 45 \\
Crush injuries & 1 \\
Total & 309 \\
\hline
\end{tabular}

*The most severe injury for each limb was listed as the indication. The amputations are traumatic injuries in this table and not surgeries or morbidities.
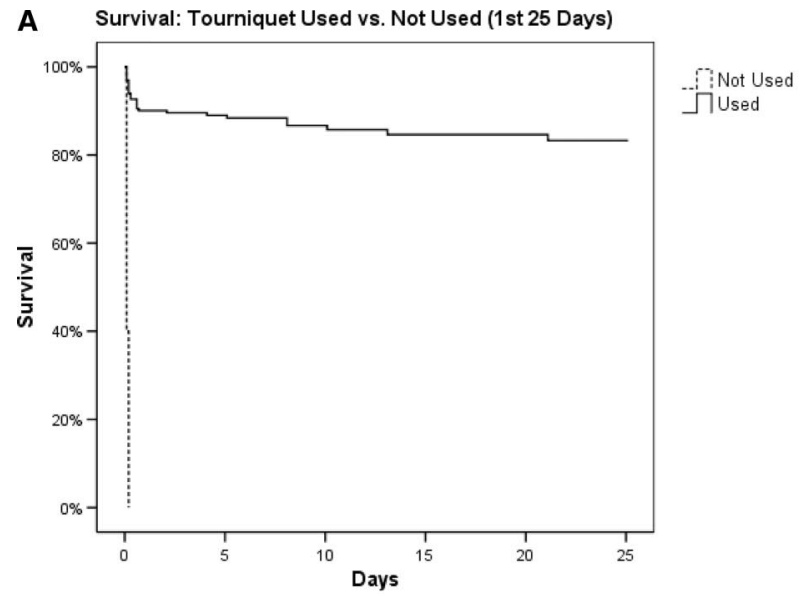

B

extricated from vehicle crashes or transported before they died. Tourniquets were indicated for them by medics, but no tourniquets were available (not brought on the trip), accessible (packed away in cargo bins), or site commanders decided to extricate or transport (scoop and go) before providing hemorrhage control (stay and play) mistakenly thinking that the patient still had time or that it was more important to first extricate or transport than first control hemorrhage. The 5 bled out in the presence of fellow soldiers often in aftermath of an explosion, lost their pulse often within a few minutes, died prehospital, and arrived at the hospital without vital signs often within 10 or 15 minutes from wounding. Some field witnesses reported that active external bleeding stopped after exsanguination, and they had underestimated the speedy lethality of uncontrolled

B Survival: No Shock vs. Shock Tourniquet Use (1st 25 Days)
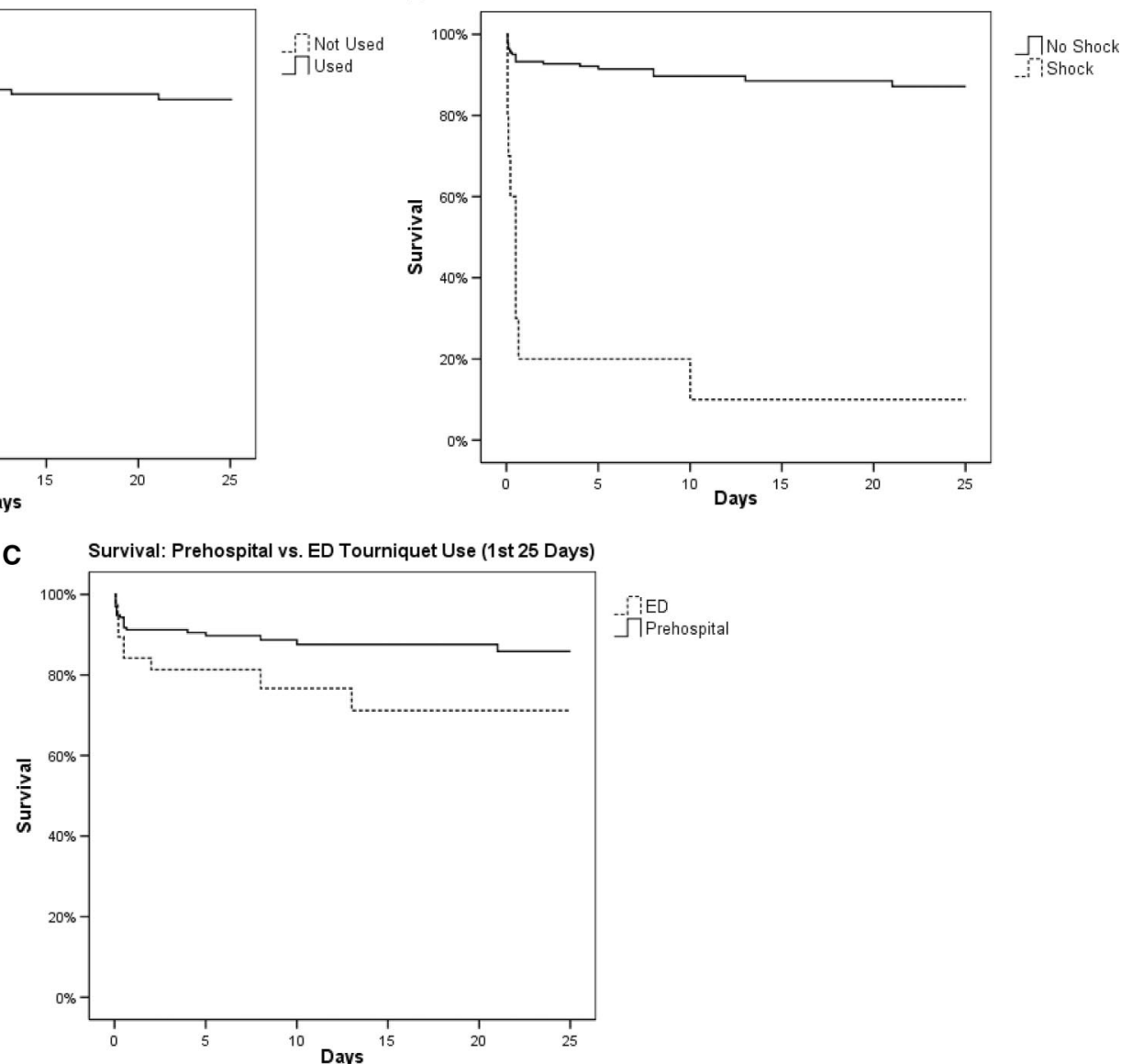

FIGURE 2. A, Survival rates for patients with tourniquets used versus not used. The Kaplan-Meier survivorship graph shows that the $92 \%$ survival rate for the patients with tourniquets used is significantly higher than the $0 \%$ survival rate for those who had tourniquets not used $(P<0.001)$. Also, the times to death are longer for those with tourniquets used. Death without tourniquets used occurred within a few minutes to 2 hours of injury. In both groups, all deaths occurred within the first 21 days. See text for matching method for these patients with only limb injuries. The groups were matched for having limb injuries only, injury severity scores (ISS), and abbreviated injury scales (AIS). B, Survival rates for patients with tourniquets used with shock present versus absent at application time. The Kaplan-Meier survivorship graph shows that the $90 \%$ survival rate for the patients with tourniquets used when shock was absent at application time is significantly higher than the $10 \%$ survival rate for those who had tourniquet used when shock was present $(P<0.001)$. Also, the times to death are longer when shock was absent. The shock present group had a higher mean injury severity scores $(P=0.04)$. $C$, Survival rates for patients with tourniquets used prehospital versus ED. The Kaplan-Meier survivorship graph shows that the $89 \%$ survival rate for the patients with tourniquets used prehospital is significantly higher than the $76 \%$ survival rate for those who had a tourniquet first used on a limb in the emergency department (ED; $P=0.05)$. Also, the average time to death is longer with those with tourniquet used prehospital. The mean injury severity scores of the 2 groups were similar $(P=0.6)$. 
TABLE 4. Tourniquets Used Versus Not Used for Casualties With Matched Injuries Only to Limbs

\begin{tabular}{lclccl}
\hline Case & Age (yrs) & \multicolumn{1}{c}{ Sex } & AIS & ISS & Alive/Dead \\
\hline 1 & 37 & Male & 4 & 16 & Dead \\
2 & 46 & Male & 4 & 16 & Dead \\
3 & 50 & Male & 4 & 16 & Dead \\
4 & 48 & Male & 4 & 16 & Dead \\
5 & 22 & Male & 4 & 16 & Dead \\
Average \pm SD & $41 \pm 11.5$ & $100 \%$ Male & 4 & 16 & $0 \%$ Alive \\
1 & 22 & Male & 4 & 16 & Alive \\
2 & 24 & Male & 4 & 16 & Dead \\
3 & 22 & Male & 4 & 16 & Alive \\
4 & 22 & Male & 4 & 16 & Alive \\
5 & 21 & Male & 4 & 16 & Alive \\
6 & 35 & Male & 4 & 16 & Dead \\
7 & 23 & Male & 4 & 16 & Alive \\
8 & 29 & Male & 4 & 16 & Alive \\
9 & 27 & Male & 4 & 16 & Alive \\
10 & 35 & Male & 4 & 16 & Dead \\
11 & 27 & Male & 4 & 16 & Alive \\
12 & \pm .5 & $100 \%$ Male & 4 & 16 & $77 \%$ Alive \\
13 & & & 4 & 16 & Alive \\
Average \pm SD & 27 & Male & 4 & 16 & Alive \\
\hline & & & &
\end{tabular}

TABLE 5. Alive/Dead Analysis of Patients by Prehospital Versus ED Tourniquet Use and Shock Presence or Absence at the Time of Application

\begin{tabular}{lccr}
\hline & \multicolumn{2}{c}{ Shock } & \\
\cline { 2 - 3 } & Absent & Present & Sum \\
\hline Tourniquet application & & & \\
Prehospital & $171 / 17$ & $1 / 5$ & 194 \\
ED & $29 / 5$ & $0 / 4$ & 38 \\
Sum & 222 & 10 & 232 \\
\hline
\end{tabular}

Numerators are the number of patients that lived, and denominators are the number of patients that died. Tourniquet use in the absence of shock was associated strongly with survival $(P=0.00000004)$, and prehospital use was associated with survival $(P=0.06)$.

limb bleeding. Hospital providers confirmed deaths and stored the dead in the morgue.

These 5 were matched with 13 patients from those 78 with only limb injuries (AIS $\geq 3$ ) of the study group's 232 patients. Matching was done for $\mathrm{AIS}^{4}$ and ISS. ${ }^{16}$ The 2 groups were statistically similar except for age and mortality; the group without tourniquets was older $(41 \pm 11.5$ years compared with $27 \pm 5.5$ years, respectively). All 5 patients without tourniquet use had only 1 limb injured, whereas 7 of 13 patients with tourniquet use had 2 limbs with tourniquets and 1 of 13 had 3 limbs with tourniquets. Tourniquet use was associated with better survival rates $(77 \%$ [10/13] vs. $0 \%$ [0/5], $P<0.007$; Figs 2, 3).

\section{Survival Rate Was Higher if Shock Was Absent Before Tourniquet Use Than if it Was Present}

All-cause mortality was $13 \%(31 / 232)$ in this study. For all deaths, the primary cause was the injury, and the secondary cause was hemorrhage with no deaths attributed to tourniquet use (Fig. 2).

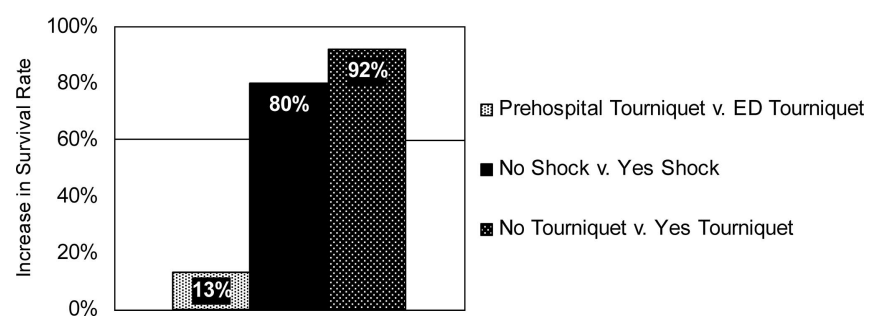

FIGURE 3. Increase in survival rate by tourniquet use. By breaking down, the tourniquet use by whether the patient was prehospital or ED, whether there was shock present or absent at the time of application, and whether tourniquets were used or not, a comparison of raw differences in survival rates indicates that the survival benefit to tourniquet use is more strongly related to tourniquet use before the patient has progressed to shock than to prehospital use.

When analyzing tourniquet use in relation to a tactical definition of shock (weak or absent radial pulse), 10 patients had tourniquet use after shock onset of which 9 died ( $90 \%)$, and 222 patients had shock absent before tourniquet use of which 22 died $(10 \% ; P<0.001$; Table 5).

When analyzing ED versus prehospital tourniquet use, 22 of 194 prehospital patients died (11\% mortality), whereas 9 of 38 ED patients died ( $24 \%$ mortality; $P=0.05)$. The halving of the mortality rate with prehospital use was associated with a $16 \%$ relative improvement in survival rate $([88.7 \%-76.3 \%] / 76.3 \%)$, whereas the mean ISSs of the prehospital and ED groups were not statistically different $(P=0.6)$.

\section{Survival Association When Shock Was Absent Before Tourniquet Use Was Strong}

The raw improvements of survival rate with tourniquet use versus no use $(92 \%)$, with shock absent versus present $(80 \%)$, and with prehospital versus ED use $(13 \%)$, indicate that most of the improvement is from use before the onset of shock (Fig. 3). Additionally, the following contingency tests indicate that shock prevention is important:

- Given prehospital use, shock absence (vs. presence) was associated with survival $(P<0.0001)$.

- Given ED use, shock absence (vs. presence) was associated with survival $(P=0.0017)$.

- Given shock absence, prehospital versus ED use was not associated with survival $(P=0.3)$.

- Given shock presence, prehospital versus ED use was not associated with survival $(P=1.0)$.

- Regardless of shock, prehospital versus ED use was weakly associated with survival $(P=0.06)$,

- Regardless of prehospital versus ED use, shock absence was associated with survival $(P<0.0001)$.

There was a strong association between shock absence at tourniquet use and survival, and a weak association between prehospital use and survival.

\section{Palsies Were Infrequent and Transient With Tourniquet Use}

Ten nerve palsies were diagnosed of which 6 were at the level of the wound and 4 were at the level of the tourniquet. The 4 palsies at the level of the tourniquet improved in the first hour to day after release, and only 1 had mild persistence at 6-days follow-up; all 4 were Iraqi patients. All 4 of these palsies at the level of the 
tourniquet were with prehospital use. No amputations were solely due to tourniquet use. For the 10 palsy patients compared with the rest of the patients, the mean durations of tourniquet use were similar (1.2 hours vs. 1.3 hours, respectively).

\section{DISCUSSION}

\section{Tourniquets Were Strongly Associated With Saved Lives Especially When Used Before the Patient Had Shock}

The main findings of the present study are that emergency tourniquet use in our population was strongly associated with saved lives and the sooner they were used, the better the patients did, particularly if the patient did not go into shock before the tourniquet was applied. Tourniquet use was associated strongly with survival if shock was absent at the time of use, and prehospital use was associated weakly with survival. Use in shock absence was associated with more life-saving than use in shock presence. Prehospital use was associated with more life-saving than ED use. These findings are likely related to the decreased rate or volume of exsanguination and improved extremity hemorrhage control by prehospital tourniquet use. Speed of successful application was vital. The median time of 10 minutes for tourniquet application compared favorably with Lakstein et al's 15 minutes, and indicated that fielding of tourniquets to individual soldiers was appropriate. When a tourniquet is indicated, placement before extrication and transportation is advised whenever possible. ${ }^{12,25,26} \mathrm{We}$ think that most of tourniquets first placed in the emergency department should have been placed prehospital, ideally before the onset of shock. Moreover, there were 5 patients that died without tourniquets that needed them, and scoop and go decisions about extrication and transport were mistakes contrary to doctrine in that hemorrhage control first with tourniquets may have saved these five. Awareness that casualties can exsanguinate rapidly to death within a few minutes from isolated, compressible limb injuries should be emphasized in training, although most casualties bleed more slowly. The reason why the shock absent versus present tourniquet use had a greater treatment effect than prehospital versus ED use was that shock onset is physiology-based, whereas the prehospital versus ED is geography based. Before this study, the only first aid device carried by medics we know of that data showed improved survival in limb injured patients was the Thomas splint. ${ }^{27}$ This splint was felt to decrease mortality by controlling extremity hemorrhage in prehospital casualties in World War I, ${ }^{28}$ which makes the splint and the tourniquet analogous. Similar to Thomas splints, the use of tourniquets improved prehospital survival, and prehospital use is needed to prevent shock onset.

\section{Current Study Compares Favorably to Other Tourniquet Studies}

The current study is the largest study to date in terms of the number of patients and tourniquets used, and our data is concordant with the experience Wolf and Adkins, ${ }^{25}$ and Lakstein et al, ${ }^{12}$ but the present study advances knowledge by measuring substantial survival benefits and limited morbidity risks. In 2003, Lakstein et al ${ }^{12}$ reported a retrospective study from Israel of 91 patients (110 limbs, 110 tourniquets, 3 models of tourniquets) and had no deaths and few data comparisons. We saw tourniquet use rates 29 times that of Lakstein et al, and the average limb AIS of the present study was higher. Lakstein et al were criticized by Husum et $\mathrm{al}^{17}$ for being unable to show that tourniquets were useful, lifesaving, or worth the risk. Further, our study was diverse as Lakstein et al had 1 nationality, all men, no children, no elderly, and no civilians and had only prehospital tourniquets of fewer types. Beekley et $\mathrm{al}^{2}$ summarized a retrospective review from the same hospital as the current study, at the beginning of widespread tourniquet implementation. They had 67 tourniquet patients and found an additional 4 of 7 patients that died without tourniquets yet were potentially salvageable. The most important factor in improving survival in our patients was attaining rapid hemorrhage control with an appropriately placed tourniquet, thus limiting risk of the negative effects of shock, and the current study provides the largest dataset of the greatest depth regarding emergency tourniquet use. Effectiveness of the tourniquets was demonstrated by survival benefits relative to no use (important to policy makers), to late use (important to prehospital providers), and to survival time (important to resuscitating providers).

\section{Morbidity Risks From Tourniquet Use Was Limited in the Current Study}

Historically, there are many anecdotes of complications associated with tourniquet use such as palsies and amputation shortening. ${ }^{1,8,16,26,29}$ However, there are only 2 retrospective cohort studies on emergency tourniquet use. ${ }^{12,25}$ In the present study, the benefits far outweighed the risks with no limbs lost solely from tourniquet use. We estimated 31 lives saved by using prehospital tourniquets compared with restricting use to $\mathrm{ED}$, and prehospital fielding of tourniquets appears plainly justified as it is evidenced to be more lifesaving than hospital use. Currently, no better alternative on the battlefield is proven for stopping bleeding in major limb trauma than tourniquets. Tourniquets are a temporary measure allowing effective hemorrhage control and should be applied before shock to save lives. We recommend a policy of encouragement of emergency tourniquet use when both the risk of lethal limb hemorrhage is high and there is systematic training of all personnel regarding appropriate tourniquet use. These conditions are met in the military setting. Policies of discouragement of tourniquet use may be appropriate in other settings, but in the current war may increase the death rate. We found minor morbidity with emergency tourniquet use, and that is detailed in another work. ${ }^{1}$

Limitations of the present study include its specific combat casualty setting and population, which may hamper generalization to the civilian trauma population. The study site, a combat support hospital, had an epidemic of severely injured coagulopathic casualties, a successful track record of performance improvement projects including emergency tourniquet use, providers rapidly exposed to large numbers of emergency tourniquets, and clinician-scientists with expertise with emergency tourniquets. Hospitals with fewer patients at risk, with providers with less familiarity, investigators with less expertise, practicing in systems without doctrine, and without lay training, may be expected to find suboptimal use and outcomes. Thus, the findings of the current study have limited generalizability until follow on studies indicate the findings are consistent over time with different patients and providers and investigators. Some readers may wish for scientific evidence of greater rigor and scope than this, the broadest and deepest work of emergency tourniquet use, but clinical survey with subgroup analysis is currently the most ethical design. Our definition of massive transfusion was 10 blood units and was not restricted to 10 packed red blood cells units as other studies have done, and this was part of our protocol authored by an orthopedist wherein the protocol idiosyncrasy was not identified or corrected at the time. We did not limit the transfusions to the first 24 hours as resuscitations often were fine-tuned after this point but by little amounts. This limitation impairs comparability among studies of different patients with different injuries and treatments. Operational hemorrhagic shock definitions are difficult and have limitations especially prehospital. None is more valid than as chosen.

Future research needs include detailed studies of morbidity, effectiveness, and other populations, other sites, and more follow up. 
We followed patients in the US system for some time, and all tourniquet-related problems occurred early, none were detected late, the last death was at 21 days from multiorgan failure, although we noted nontourniquet problems such as flap failure months later with good follow-up. The patients that needed tourniquets but did not get them need an education effort aimed at soldiers regarding the life-saving capacity of tourniquets if used well promptly. Repeated, consistent studies with detailed analysis can help change how we think about resuscitative measures for severely injured limb casualties, and well-designed observational surveys can fill specific knowledge gaps ethically in the foreseeable future.

\section{ACKNOWLEDGMENTS}

The authors thank Joanna G. Branstetter, MD, (Research Fellow, USA ISR) and Amy Newland (Editor, USA ISR) for assistance in manuscript preparation, Sumeru G. Mehta, MD, (Emergency Physician, Brooke Army Medical Center) for the number of admissions, and John Jones for contingency testing.

\section{REFERENCES}

1. Kragh JF Jr, Walters TJ, Baer DG, et al. Practical use of emergency tourniquets to stop bleeding in major limb trauma. J Trauma. 2008;64(suppl): S38-S49; discussion S49-S50.

2. Beekley AC, Sebesta JA, Blackbourne LH, et al. Prehospital tourniquet use in Operation Iraqi Freedom: effect on hemorrhage control and outcomes. J Trauma. 2008;64(supp1):S28-S37; discussion S37.

3. Bellamy RF. The causes of death in conventional land warfare: implications for combat casualty care research. Mil Med. 1984;149:55-62.

4. Holcomb JB, McMullin NR, Pearse L, et al. Causes of death in U.S. special operations forces in the global war on terrorism: 2001-2004. Ann Surg. 2007;245:986-991.

5. Holcomb J, MacPhee M, Hetz S, et al. Efficacy of a dry fibrin sealant dressing for hemorrhage control after ballistic injury. Arch Surg. 1998;133:32-35.

6. Calkins D, Snow C, Costello M, et al. Evaluation of possible battlefield tourniquet systems for the far-forward setting. Mil Med. 2000;165:379-384.

7. Mabry RL, Holcomb JB, Baker AM, et al. United States Army Rangers in Somalia: an analysis of combat casualties on an urban battlefield. J Trauma. 2000;49:515-528; discussion 528-529.

8. Walters TJ, Mabry RL. Issues related to the use of tourniquets on the battlefield. Mil Med. 2005;170:770-775.
9. Wenke JC, Walters TJ, Greydanus DJ, et al. Physiological evaluation of the US Army one-handed tourniquet. Mil Med. 2005;170:776-781.

10. Mabry RL. Tourniquet use on the battlefield. Mil Med. 2006;171:352-356.

11. Walters TJ, Wenke JC, Kauvar DS, et al. Effectiveness of self-applied tourniquets in human volunteers. Prehosp Emerg Care. 2005;9:416-422.

12. Lakstein D, Blumenfeld A, Sokolov T, et al. Tourniquets for hemorrhage control on the battlefield: a 4-year accumulated experience. J Trauma. 2003;54(suppl):S221-225.

13. King RB, Filips D, Blitz S, et al. Evaluation of possible tourniquet systems for use in the Canadian Forces. J Trauma. 2006;60:1061-1071.

14. Pillgram-Larsen J, Mellesmo S. Not a tourniquet, but compressive dressing. Experience from 68 traumatic amputations after injuries from mines [English abstract]. Tidsskr Nor Laegeforen. 1992;112:2188-2190.

15. Navein J, Coupland R, Dunn R. The tourniquet controversy. J Trauma 2003;54(suppl):S219-S220

16. Welling DR, Burris DG, Hutton JE, et al. A balanced approach to tourniquet use: lessons learned and relearned. J Am Coll Surg. 2006;203:106-115.

17. Husum H, Gilbert M, Wisborg T, et al. Prehospital tourniquets: there should be no controversy. J Trauma. 2004;56:214-215.

18. Husum H, Ang SC, Fosse E. War Surgery Field Manual, Penang, Malaysia: Third World Network. 1995;85:145.

19. Watson-Jones R. Fractures and Joint Injuries. Vol 1. 4th ed. London: Livingstone; 1955:121-122.

20. Klenerman L. The tourniquet in surgery. J Bone Joint Surg Br. 1962;44(-B) 937-943.

21. Dorlac WC, DeBakey ME, Holcomb JB, et al. Mortality from isolated civilian penetrating extremity injury. J Trauma. 2005;59:217-222.

22. Sebesta J. Special lessons learned from Iraq. Surg Clin North Am. 2006;86 711-726.

23. McManus J, Yershov AL, Ludwig D, et al. Radial pulse character relationships to systolic blood pressure and trauma outcomes. Prehosp Emerg Care. 2005;9:423-428

24. PHTLS. Military. 6th ed. St Louis, MO: Mosby; 2007:501-519.

25. Wolf LH, Adkins TF. Tourniquet problems in war injuries. Bull US Army Medl Dep. 1945;87:77-84.

26. Bellamy RF. Why is Marine combat mortality less than that of the Army? Mil Med. 2000;165:362-367.

27. Gray HMW. The Early Treatment of War Wounds. London: Oxford University Press; 1919:49-63

28. Kirkup J. Fracture care of friend and foe during World War I. Aust N Z J Surg. 2003;73:453-459.

29. Klenerman L. The Tourniquet Manual. London: Springer; 2003. 